\title{
Quantum dot single-photon emission coupled into single-mode fibers with 3D printed micro-objectives
}

Cite as: APL Photonics 5, 106101 (2020); https://doi.org/10.1063/5.0014921

Submitted: 22 May 2020 . Accepted: 10 September 2020 . Published Online: 01 October 2020

Lucas Bremer, Ksenia Weber, Sarah Fischbach, Simon Thiele, Marco Schmidt, Arsenty Kaganskiy, Sven Rodt, (D) Alois Herkommer, (D) Marc Sartison, (D) Simone Luca Portalupi, (D) Peter Michler, (D) Harald Giessen, and (D) Stephan Reitzenstein

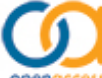

1)

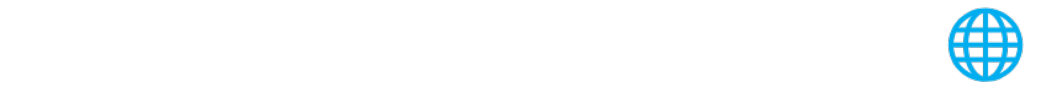

\section{ARTICLES YOU MAY BE INTERESTED IN}

Development of site-controlled quantum dot arrays acting as scalable sources of indistinguishable photons

APL Photonics 5, 096107 (2020); https://doi.org/10.1063/5.0013718

An optical chip for self-testing quantum random number generation

APL Photonics 5, 101301 (2020); https://doi.org/10.1063/5.0022526

Terahertz transient stimulated emission from doped silicon

APL Photonics 5, 106102 (2020); https://doi.org/10.1063/5.0020654

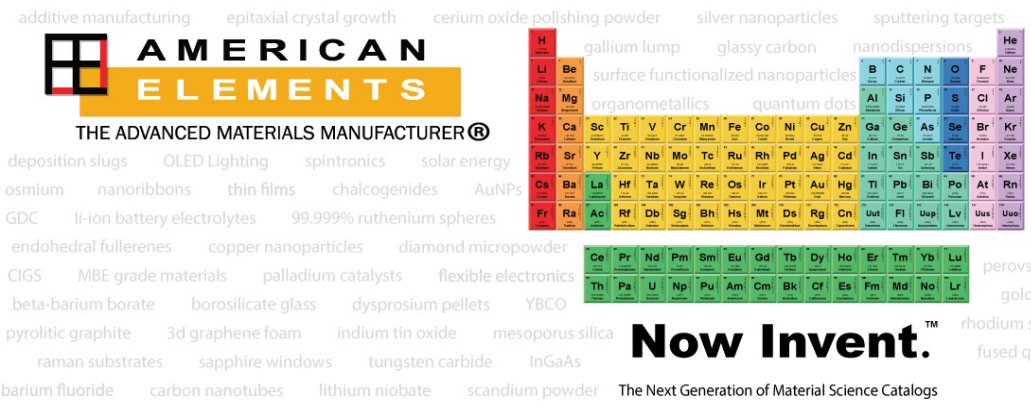

Dorsibilities so you can Now Invent!

Over 15,000 certifed high purity laboratory chemicals, metals, \& advanced materials and a state-of-the-art Research Center. Printable GHS-compliant Safety Data Sheets. Thousands of new products. And much more. All on a secure multi-language "Mobile Responsive" plattorm. 


\title{
Quantum dot single-photon emission coupled into single-mode fibers with 3D printed micro-objectives
}

\author{
Cite as: APL Photon. 5, 106101 (2020); doi: 10.1063/5.0014921 \\ Submitted: 22 May 2020 - Accepted: 10 September 2020 • \\ Published Online: 1 October 2020
}

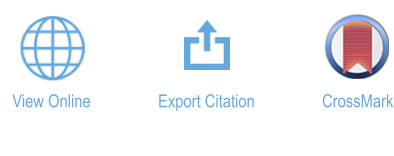

Lucas Bremer, ${ }^{1}$ Ksenia Weber, ${ }^{2}$ Sarah Fischbach, ${ }^{1}$ Simon Thiele, ${ }^{3}$ Marco Schmidt, ${ }^{1}$ Arsenty Kaganskiy, Sven Rodt, ${ }^{7}$ Alois Herkommer, ${ }^{3}$ (D) Marc Sartison, ${ }^{4}$ (D) Simone Luca Portalupi, ${ }^{4}$ (D) Peter Michler, ${ }^{4}$ Harald Giessen, ${ }^{2}$ (D) and Stephan Reitzenstein ${ }^{1, a)}$ (D)

\begin{abstract}
AFFILIATIONS
${ }^{1}$ Institute of Solid State Physics, Technische Universität Berlin, Berlin, Germany

${ }^{2} 4$ th Physics Institute and Research Center SCOPE and Integrated Quantum Science and Technology Center IQ ${ }^{\text {ST }}$. University of Stuttgart, Stuttgart, Germany

${ }^{3}$ Institute for Applied Optics (ITO) and Research Center SCOPE, University of Stuttgart, Stuttgart, Germany

${ }^{4}$ Institut für Halbleiteroptik und Funktionelle Grenzflächen, Center for Integrated Quantum Science and Technology (IQ ${ }^{\text {ST }}$ ) and Research Center SCoPE, University of Stuttgart, Stuttgart, Germany
\end{abstract}

a) Author to whom correspondence should be addressed: stephan.reitzenstein@physik.tu-berlin.de

\begin{abstract}
User-friendly single-photon sources with high photon-extraction efficiency are crucial building blocks for photonic quantum applications. For many of these applications, such as long-distance quantum key distribution, the use of single-mode optical fibers is mandatory, which leads to stringent requirements regarding the device design and fabrication. We report on the on-chip integration of a quantum dot (QD) microlens with a 3D-printed micro-objective in combination with a single-mode on-chip fiber coupler. The practical quantum device is realized by the deterministic fabrication of the QD-microlens via in situ electron-beam lithography and the 3D two-photon laser writing of the on-chip micro-objective and fiber chuck. A QD with a microlens is an efficient single-photon source, whose emission is collimated by the on-chip micro-objective. A second polymer microlens is located at the end facet of the single-mode fiber and ensures that the collimated light is efficiently coupled into the fiber core. For this purpose, the fiber is placed in an on-chip fiber chuck, which is precisely aligned to the QD-microlens thanks to the sub-micrometer processing accuracy of high-resolution two-photon direct laser writing. The resulting quantum device has a broadband photon extraction efficiency, a single-mode fiber-coupling efficiency of $22 \%$, a measured single-photon flux of $42 \mathrm{kHz}(8.9 \mathrm{kHz})$ under cw (pulsed) optical excitation, which corresponds to $1.5 \mathrm{MHz}(0.3 \mathrm{MHz})$ at the single-mode fiber output, and a multi-photon probability in terms of $\mathrm{g}^{(2)}(0)=0.00 \pm_{0.00}^{0.04}(0.13 \pm 0.05)$ under cw (pulsed) optical excitation. The stable design of the developed fiber-coupled quantum device makes it highly attractive for integration into user-friendly plug-and-play quantum applications.
\end{abstract}

(C) 2020 Author(s). All article content, except where otherwise noted, is licensed under a Creative Commons Attribution (CC BY) license (http://creativecommons.org/licenses/by/4.0/). https://doi.org/10.1063/5.0014921

\section{INTRODUCTION}

The development of real-world quantum communication networks $^{1,2}$ that offer a previously unattained level of data transfer security $^{3,4}$ has become very dynamical in recent years. In addition to the first quantum networks based on optical fibers ${ }^{5,6}$ and free-space channels, ${ }^{7,8}$ expanded solutions in the form of satellitebased quantum communication networks ${ }^{9,10}$ have also been developed. In this context, strongly attenuated pulsed lasers are still frequently used as the photon sources for decoy-state-based protocols, ${ }^{11,12}$ offering the highest transfer rates so far. However, ultimate performance can only be obtained by using true 
single-photon sources, based, for instance, on semiconductor quantum dots (QDs), which not only promise on-demand operation but also feature nowadays the purest single-photon emission of all known quantum light sources. ${ }^{13,14}$ It is noteworthy that only the sources with non-classical single-photon statistics can exploit the full potential of quantum communication. ${ }^{15,16}$ In addition, the availability of the true single-photon sources is crucial for the implementation of the quantum repeater protocol. ${ }^{1,17}$ In fact, the on-demand sources of indistinguishable and entangled photons ${ }^{18-21}$ are key to make the dream of long-distance multi-partite networks become a reality. Here, it is important to ensure that the photons always propagate in the same defined spatio-temporal mode, ${ }^{22}$ making the use of single-mode fibers (SMFs) quantum channel indispensable.

The on-chip fiber-coupled sources have the decisive advantage of enabling ultra-stable operation without additional free-beam optics. This feature reduces the complexity of the source enormously and, as a result, boosts its flexibility and application relevance. In the past, multimode fibers ${ }^{23}$ or SMF bundles ${ }^{24}$ were used to realize the fiber-coupled single-photon sources. Only recent progress in nanofabrication techniques has paved the way for the single-modecoupling of semiconductor QDs integrated into Fabry-Perot optical microcavities, ${ }^{25}$ cylindrical mesas, ${ }^{26}$ photonic crystal nanobeams, ${ }^{27}$ or waveguide-based devices. ${ }^{28-30}$ In the present work, we take a different approach and want to combine the strength of the QDmicrolenses in terms of extraction efficiency ${ }^{31}$ with the advantages of the precise and flexible 3D two-photon direct laser (TPL) patterning of photonic microstructures. ${ }^{32-}$

A significant challenge to be tackled for the realization of efficient coupling of a QD-microlens to a SMF with a core diameter of only $4.4 \mu \mathrm{m}$ and a numerical aperture (NA) as low as 0.13 is that, although the QD-microlenses are mechanically very robust and provide the broadband enhancement of photon extraction, their emission is only moderately directed with respect to small NAs. As a result, a micro-objective design was developed that collects and collimates the outcoupled radiation of the QD-microlens. For this purpose, the approach described in Ref. 37 was revised and extended using ray-tracing calculations with Zemax. In the present work, we developed a total internal reflection (TIR) microlens, serving as the light-collection micro-objective, which is printed with sub-micrometer accuracy ${ }^{35}$ onto a QD-microlens using 3D femtosecond direct laser writing. The TIR design used here offers two major advantages over the multi-element objective applied in Ref. 37: First, it collects light from the whole hemisphere above the quantum dot. This corresponds to a numerical aperture equal to the refractive index $\mathrm{n}$ of the used photopolymer ( $\mathrm{n} \cong 1.54$ ). Compared to the best multi element design with an NA of 0.7 , this is an increase of more than a factor of two. Second, the TIR lens strongly reduces losses at the semiconductor interface as the photo resist moderates the transition from GaAs to air and the number of polymer to air interfaces is reduced from four to only one. A further advantage is that the TIR lens is more compact and faster to fabricate. The main disadvantages are that the alignment needs to be much more precise $(<100 \mathrm{~nm})$ in comparison to that in Ref. $37(<5 \mu \mathrm{m})$ and more external strain is induced at low temperatures. Further details on the lens design can be found in Ref. 38.

Additionally, similar to Ref. 39, we designed and realized an in situ TPL-printed on-chip fiber chuck aligning the
QD-microlens-system to a coupling lens on the face facet of a SMF. Lensed fibers have proven to be highly tolerant to displacement along the fiber's lateral axis in a collimated beam setup with respect to the coupling efficiency. ${ }^{40}$ The synergetic combination of all these components results in a precise, fully integrated, and ultrastable micro-optical on-chip photonic system for applications in fiber-based quantum communication.

\section{DEVICE TECHNOLOGY AND FABRICATION}

The sample is based on a wafer heterostructure consisting of InGaAs QDs grown on a (100) GaAs substrate by metal-organic chemical vapor deposition. A back-side distributed Bragg reflector (DBR) consisting of $23 \mathrm{GaAs}(67 \mathrm{~nm}) / \mathrm{Al}_{0.9} \mathrm{Ga}_{0.1}$ As $(78 \mathrm{~nm}) \lambda / 4$ thick layer pairs is located underneath the QD layer at a distance of $67 \mathrm{~nm}$ to reflect the light emitted into the lower hemisphere, and thus, increase the photon-extraction efficiency normal to the sample surface. The self-assembled QDs are randomly distributed in position and wavelength with a center wavelength of around $920 \mathrm{~nm}$. Above the QD layer, a $420 \mathrm{~nm}$ thick capping layer is grown, which is required for the deterministic structuring of the QD-microlenses by $3 \mathrm{D}$ in situ electron-beam lithography (EBL). With the help of the numerical optimization of the microlens design, it is possible to achieve outcoupling efficiencies of almost $30 \%$ for an NA of $0.4 .^{31,41}$

For the fabrication of the QD-microlenses, a $80 \mathrm{~nm}$ thick electron-sensitive CSAR62 resist film ${ }^{42}$ is first spin-coated onto the sample, and then a specially modified electron scanning microscope is used to record cathodoluminescence maps at $\mathrm{T}=10 \mathrm{~K}$. Suitable QDs are selected based on the emission wavelength and the emission intensity of the excitonic lines, and a lenticular dose profile is then introduced into the resist at the positions of selected QDs. In the later anisotropic, plasma-enhanced reactive ion etching step, the electron-sensitive resist remaining on the sample surface after exposure and development acts as an etching mask so that the introduced lens profile is transferred into the semiconductor material, resulting in the monolithic QD microlenses.

To realize a high photon coupling efficiency into a SMF, the radiation pattern of the QD-microlens must be taken into account, i.e., to maximize the usable photon flux, the emission should be collected from the largest possible solid angle and coupled into the fiber. We solve this issue by a configuration consisting of two TPL written polymer micro-optical elements, as illustrated in Fig. 1(a). A total-internal-reflection micro-objective on the QD-microlens is used to collimate its divergent emission, while an NA matched (NA $=0.13$ ) coupling microlens on the single mode fiber is used to focus the beam down onto the fiber core. One advantage of this optical system is that it is rather insensitive to the distance between TIR and coupling lenses, as long as the beam can still be regarded as collimated. Details on the design of the TPL written lenses, its optimization, and its relationship to the achievable photoluminescence enhancement are given in the supplementary material and in Ref. 38. For these optimization measurements, high-precision low temperature deterministic lithography was used ${ }^{43}$ marking the emitter for further room temperature fabrication. ${ }^{35,44}$ Furthermore, a first characterization of the fiber in-coupling performances was carried out in a high stability cryostat where the position of the fiber with respect to the sample can be precisely controlled (see the 
a)

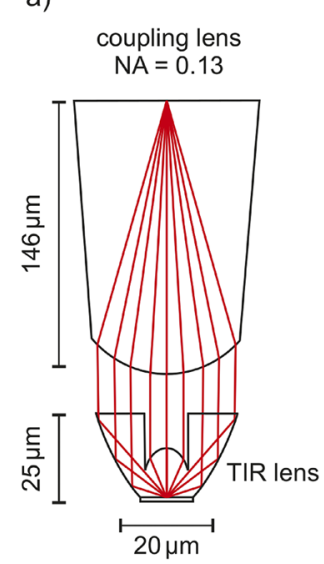

f)

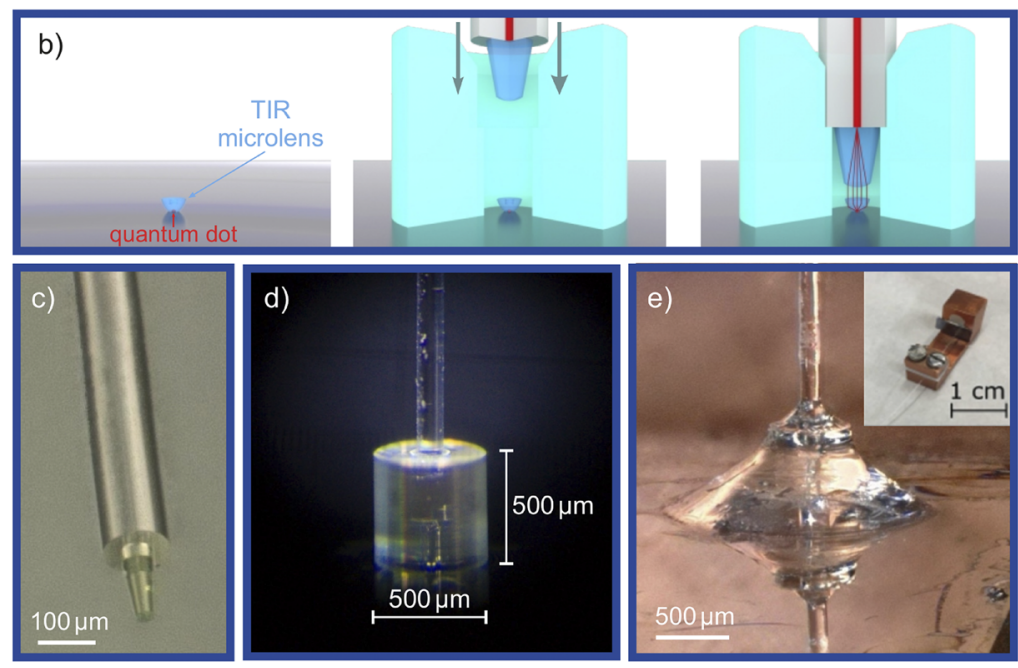

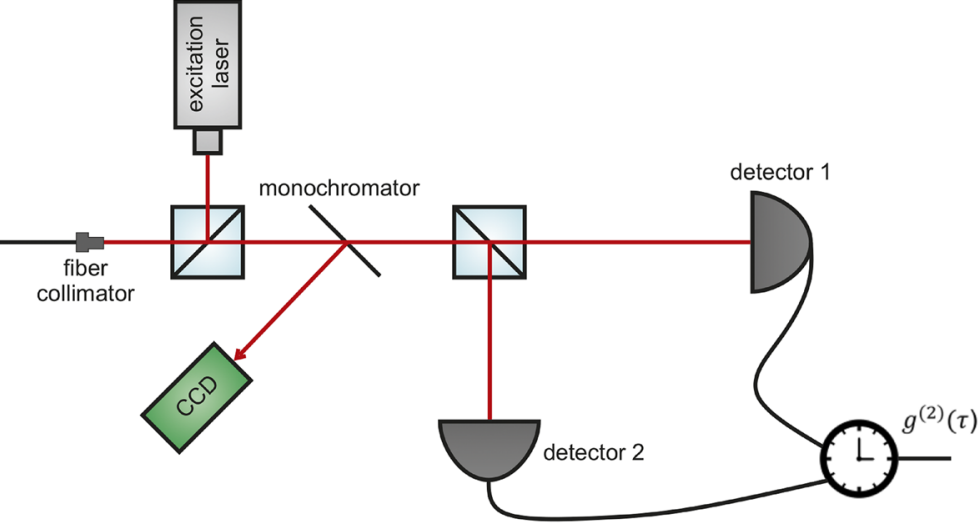

FIG. 1. (a) Optical design for coupling light from a QD-microlens into a single-mode fiber obtained from sequential ray tracing. Light that is emitted from the QD-microlens is collimated by a TIR lens (output NA $=0.001$, see the supplementary material) and focused onto the fiber core by an NA matched (NA $=0.13)$ spherical focus lens. (b) Scheme showing the fabrication of the integrated fiber coupled single-photon source. The TIR lens is printed onto the QD-microlens (left), followed by 3D printing the on-chip fiber chuck and inserting the SMF with the focus lens printed to its end facet (middle). After inserting the fiber, the light from the QD-microlens is efficiently coupled into the fiber core via the micro-optical system (right). (c) Microscope image of a SMF (THORLABS 780-HP) with an NA matched focus lens printed onto it. (d) Microscope image of an optical SMF inserted into a fiber chuck via a manual XYZ-flexure stage. (e) Microscope image of a SMF inserted into a fiber chuck and glued to the substrate with UV-cured glue. Inset: fiber-coupled QD-sample mounted onto a strain-relief copper holder for mounting in a cryostat. (f) Scheme for the second-order autocorrelation measurement. The fiber-coupled sample is inserted into a liquid helium can and excited via an off-resonant excitation laser. The fiber-coupled QD signal is then spectrally analyzed and sent to a Hanbury Brown and Twiss setup to obtain the time-correlated signal.

supplementary material). A maximum fiber-coupling efficiency of $26 \% \pm 5 \%$ was observed. This motivated the fabrication of the fiber chuck to replicate this performance in a mechanically stable manner. Controlling the reciprocal position further showed that in the transverse direction, precise alignment with sub-micrometer accuracy of all components is required. ${ }^{33,34}$ The micro-optics are fabricated from IP-Dip photoresist ${ }^{45}$ with a commercial femtosecond two-photon 3D laser printer (Photonics Professional GT, Nanoscribe $\mathrm{GmbH}$ ). The laser beam is scanned in the lateral direction by two galvanometric mirrors through a $63 \times$ microscope objective in dip-in configuration (NA $=1.4)$.

In the case of the TIR lens, the photoresist is applied onto the semiconductor sample and the objective is immersed into it, while for the fiber lens, the photoresist is applied directly onto the objective and the fiber is inserted into it subsequently. In the axial direction, the semiconductor sample is moved by a piezoelectric crystal, while the fiber is left stationary and the objective is moved instead.

The TIR lens is aligned to the QD-microlens using prefabricated alignment markers. As the TIR lens will cover the etched markers after its fabrication, new 3D printed markers of the same shape are printed along with the TIR lens. The SMF core is located by shining light from a red LED onto the other end facet of the fiber, which leads to the fiber core lighting up in the microscope image for precisely placing the coupling lens (more details can be found in Ref. 46). The total printing time for the TIR lens was about $5 \mathrm{~min}$. Due to its substantially larger volume, the coupling lens took about $1 \mathrm{~h}$ 
to be produced. After the printing, the structures were developed in mr-Dev 600 (micro resist technology $\mathrm{GmbH}$ ) for several minutes. During this process, the unexposed photoresist is removed. After pre-characterizing the QD-microlenses with the TIR lenses printed onto them by micro-PL spectroscopy [see Fig. 2(a)], fiber chucks are fabricated around the lenses using the $3 \mathrm{D}$ printed markers for alignment. Due to the large size of the fiber chuck, the polymer IP-S, which is a lower resolution photoresist and an objective with a larger field-of-view $(25 \times, \mathrm{NA}=0.8)$ are used in this processing step. The printing time for the fiber chuck is roughly $45 \mathrm{~min}$. After development, the fiber integration is performed. For this, the SMF with the coupling lens printed on top [shown in Fig. 1(c)] is mounted onto a
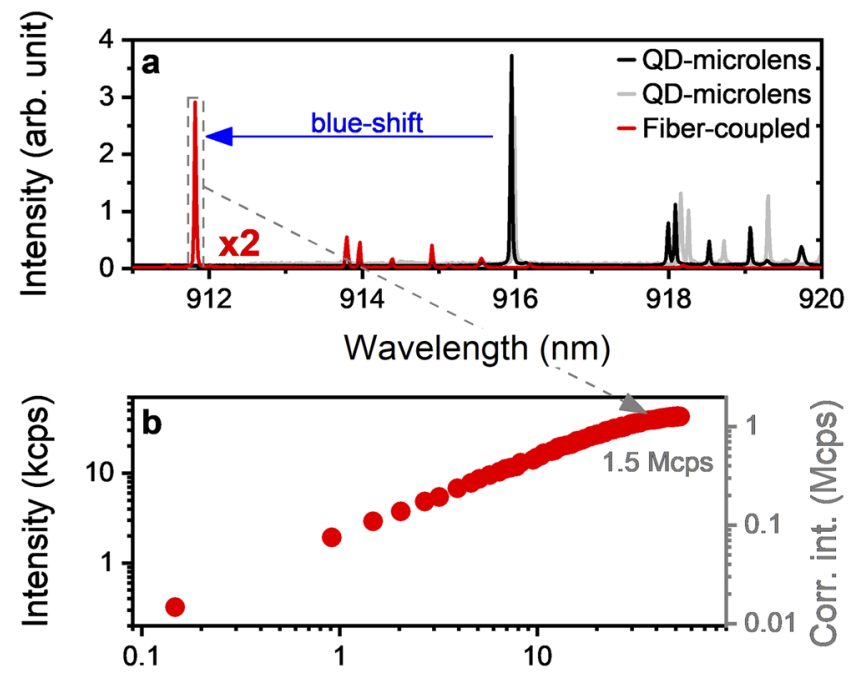

Excitation power $(\mu \mathrm{W})$

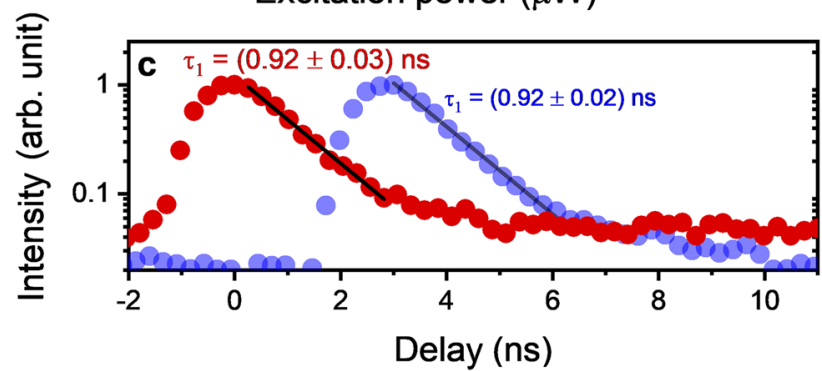

FIG. 2. (a) micro-PL spectrum of the QD-microlens before (black) and after (red) SMF-coupling. The induced compressive strain of the micro-objective causes a blue-shift of the QD emission lines of about $4 \mathrm{~nm}$. The spectrum of the fibercoupled sample is shown with the doubled intensity for better comparability. In gray, an additional spectrum of the QD-microlens is shown, which was generated during the optimization of the micro-objective geometry. It reveals that the blueshift is reversible by removing the micro-objective. (b) Single-photon rate under the $\mathrm{CW}$ optical excitation of the QD emission line marked in (a). For the right $y$ axis, the setup efficiency was taken into account and the photon flux detected by a SPCM was corrected accordingly. (c) Measured lifetime for the QD line from (a) (red). The lifetime $\tau_{1}=(0.92 \pm 0.03)$ ns corresponds to the inverse of the slope of a linear fit to the falling edge of the pulse. A lifetime measurement taken during the pre-characterization of the QD microlens yields $\tau_{1}=(0.92 \pm 0.02) \mathrm{ns}$ and is shown in blue (horizontally shifted for clarity). The lifetime is not influenced by the fiber-coupling. manual XYZ-flexure stage and inserted into the fiber chuck under a microscope. A mirror angled at $45^{\circ}$ is used to monitor the position of the fiber relative to the fiber chuck opening in all directions.

A sketch of the fiber integration is shown in Figs. 1(b) and a microscope image of the inserted fiber in Fig. 1(d). The fiber in its chuck is then fixed to the substrate by depositing a small droplet of UV-cured glue with another piece of the optical fiber mounted onto a second XYZ-flexure stage. To avoid the fiber chuck to detach from the substrate when cooled down to cryogenic temperatures, the UV glue is spread to cover the entire fiber chuck [see Fig. 1(e)]. The sample is then mounted onto a copper holder with a built-in strain relief, as shown in the inset of Fig. 1(e). The substrate is glued to the bottom of the holder with a conductive silver paste and the SMF is fixed about $1 \mathrm{~cm}$ away from it between two pieces of polytetrafluoroethylene (Teflon) with two small screws. This makes the device highly robust and easy to ship and allows it to be mounted in a specially designed sample holder located at the lower end of a dip stick. The dip stick is inserted into a user-friendly standard helium $(\mathrm{He})$ transport vessel so that the fiber-coupled device can be studied with the setup shown in Fig. 1(f).

\section{RESULTS AND DISCUSSION}

In the following, we discuss the optical properties of the QDmicrolens and that of the complete fiber-coupled single-photon source. To quantify the fraction of QD photons emitted by the QD-microlens and coupled into the SMF, the micro-PL spectra were recorded before and after processing of the TIR objective and subsequent fiber coupling. Figure 2(a) shows a microPL spectrum of a QD-microlens before the processing of the TIR objective (black trace). The setup shown in Fig. 1(f) was used, except that the sample was placed in a He-flow cryostat instead of immersing it directly in liquid helium. Furthermore, a microscope objective with an NA of 0.65 was used for the measurement. In the micro-PL spectrum, the bright trion line at $916 \mathrm{~nm}$ is particularly noticeable besides the neutral excitonic complex around $918 \mathrm{~nm}$. The excitonic states were identified by polarization and excitation power dependent micro-PL measurements. In addition, the time-resolved measurements were used to confirm the assignment based on the decay constants of the excitonic states. Due to the highest micro-PL intensity, the trion line was chosen for the more detailed investigations in the following, including photonautocorrelation measurements to confirm the quantum nature of emission.

Figure 2(a) shows in red the spectrum of the same QDmicrolens under comparable excitation conditions, i.e., close to saturation, after SMF coupling. Each micro-PL spectrum is normalized by the setup efficiency (He-flow cryostat configuration: $5.1 \% \pm 0.5 \%$, fiber-coupled configuration: $2.8 \% \pm 0.3 \%$ ) to ensure the comparability of the intensities. In addition, the intensity of the fiber-coupled spectrum has been doubled to increase visibility. It is apparent that the fiber-coupling of the QD-microlens shifts the QD spectrum to lower wavelengths by a blue-shift of about $4 \mathrm{~nm}$, comparable to the previously observed values for the $3 \mathrm{D}$ printed hemispheric lenses. ${ }^{35}$ This blue shift was observed reproducibly for two cooling cycles. Likewise, a second QD-microlens sample, which was fiber-coupled, showed a blue shift of $4 \mathrm{~nm}$ (see the supplementary material). A 
much smaller shift of $0.2 \mathrm{~nm}$ due to the influence of a TPL patterned on-chip micro-objective was already discussed in Ref. 37. However, in the present work, we use a different design for the micro-objectives, which, in addition to the on-chip fiber chuck, may explain the larger blue-shift. In general, we have found that the different geometries of micro-objectives consistently lead to blue shifts of $0.2 \mathrm{~nm}-4.0 \mathrm{~nm}$, with a tenuous correlation between the lens diameter and the resulting wavelength shift-the larger the diameter, the smaller the blue shift. The blue-shift is attributed to the compressive stress of the semiconductor material including the QD caused by the printed micro-objective. ${ }^{35}$ The relationship of the blue shift to the polymer structure on top of the sample is also justified by the fact that the wavelength shift is reversible, i.e., the QD spectrum red-shifts to the original position after the removal of the micro-objective and the fiber chuck [gray trace in Fig. 2(a)]. This was observed when optimizing the process flow iteratively, whereby different objective geometries were printed on a variety of QDmicrolenses whose emission properties were monitored after each processing step. However, the basic optical properties of the QD do not seem to be influenced by this strain effect. The linewidth of $<25 \mu \mathrm{eV}$ is still limited by the resolution of the spectrometer $(25 \mu \mathrm{eV})$ and the lifetime [Fig. 2(c)], and the multi-photon probability (Fig. 3) is not affected.

Besides the blue-shift, it is also noticeable that the continuous background of the spectrum is significantly reduced by more than $2 / 3$ due to the fiber-coupling, and thus, a clear and background-free spectrum can be observed from the fiber-coupled QD-microlens. This is very advantageous and is probably because the single-mode fiber facet acts effectively as a local pinhole, thereby selecting the emission of the target QD and suppressing contributions from
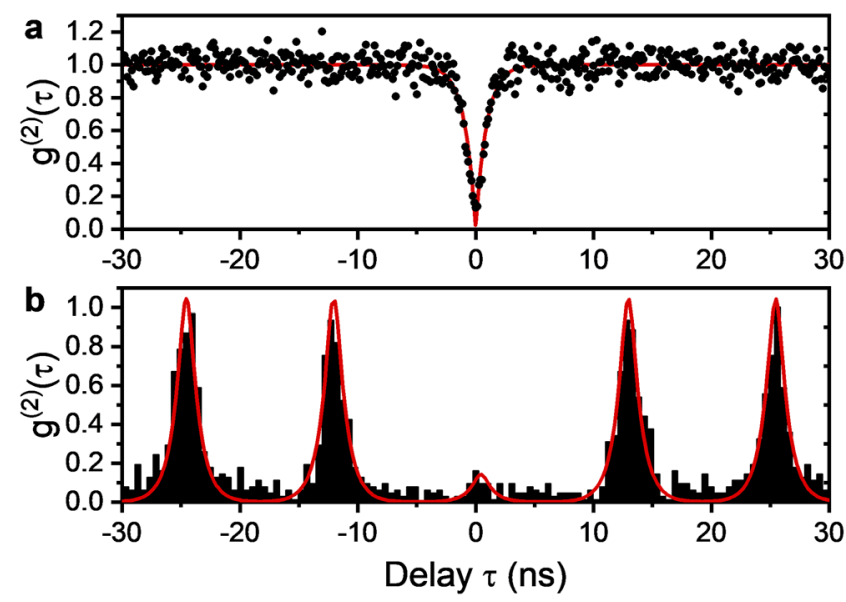

FIG. 3. Measured second-order autocorrelation function. The histograms show single-photon emission with low multi-photon probability of the fiber-coupled device. (a) Normalized histogram for non-resonant $\mathrm{cw}$ excitation. The red solid line shows an exponential fit of the measured data without the consideration of the time resolution of the setup (without deconvolution). This results in a value of $g^{(2)}(0)=0.00 \pm_{0.00}^{0.04}$. (b) Photon autocorrelation histogram for non-resonant pulsed excitation at $80 \mathrm{MHz}$. The red line represents a fit to the data, assuming a mono-exponential radiative decay of $\tau_{1}=0.92 \mathrm{~ns}$ and considering the overall time resolution of the HBT setup of $495 \mathrm{ps}$. In this approach, one obtains a value of $g^{(2)}(0)=0.13 \pm 0.05$. the wetting layer and possibly other non-intentionally integrated QDs centered at other positions and wavelengths. Very clean QD emission spectra are of particular interest for the use of the fibercoupled device in stand-alone applications, where for practical reasons, narrow fiber-coupled optical filters are used instead of bulky and expensive monochromators. ${ }^{26}$ These filters have usually a higher bandwidth than the state of the art monochromators, which could increase multi-photon events.

To determine the coupling efficiency into the single-mode fiber, which is coupled and glued to the chip, we compare the integrated spectral area of the trion line before and after the processing. It should be noted that the fiber-coupling efficiency is based on the comparison of the trion intensity detected with a microscope objective with an NA of 0.65 of the pure QD microlens without TIR lens compared to the full fiber-coupled lens system. To account for the different NAs under which the light was collected in both measurements, the value for the reference measurement before fibercoupling is increased by a factor of $1.17 \pm 0.01$. This correction is based on the numerical simulations of the QD microlenses, ${ }^{31}$ which show that the photon extraction efficiency increases slightly from $22.6 \%$ at $\mathrm{NA}=0.65 \%$ to $26.4 \%$ at $\mathrm{NA}=1.0$. This comparison yields an in-coupling efficiency from the QD-microlens into the fiber of $22 \% \pm 2 \%$, which reflects the excellent performance of the matched lens geometries despite the non-ideal far-field pattern of the QDmicrolens. If no lens system is used to focus the collimated beam into the fiber core, a coupling efficiency of $<1 \%$ would have to be expected. However, according to our ray tracing model and the fiber coupling efficiency tool from Zemax/OpticStudio, a coupling efficiency of $81.8 \%$ should theoretically be possible for the present design. However, it should be noted that the ray tracing model simplifies the situation and, among other things, does not take into account the non-angular-isotropic radiation characteristic of the QD-microlens, instead it assumes it as a point source due to its small diameter compared to the TIR lens. This has a strong influence on the expected fiber-coupling efficiency, which is overestimated in the ray tracing simulation since this model does not take into account the resulting non-ideal mode overlap of the field distribution with the Gaussian mode of the SM fiber. Furthermore, the dielectric losses due to reflections at material interfaces were not considered, which we estimate to amount to at least $24 \%$. It is noticeable that in addition to the sample shown, another QD-microlens was fibercoupled, resulting in a comparable value for the in-coupling efficiency from the QD-microlens into the fiber of $24 \% \pm 2 \%$ (see the supplementary material). In addition, in the validation of the coupling system, ${ }^{38}$ the coupling efficiency of $26 \% \pm 5 \%$ was measured, which is a good value for low-dimensional single-mode fiber coupling, even if there is a large discrepancy to the simplified ray tracing model mentioned above. We expect to reach significantly higher fiber-coupling efficiencies in the future, e.g., by using, for instance, near-field coupling in the circular Bragg grating single-photon sources.

If the trion line of the spectrum shown in Fig. 2(a) is spectrally selected with a monochromator and detected with an avalanchephotodiode-based single-photon counting module (SPCM), we achieve a count rate of $42 \mathrm{kHz}$ at saturation under $\mathrm{cw}$ excitation at $532 \mathrm{~nm}$ for this fiber coupled configuration. Considering the overall transmission of our setup of $2.8 \% \pm 0.3 \%$, this corresponds to a single-photon flux of $1.5 \mathrm{MHz}$ at the end of the single-mode fiber. 
Figure 2(b) indicates the count rate also for lower excitation powers. In order to estimate the overall efficiency of the fiber-coupled device starting with the QD's emission, pulsed excitation with a repetition frequency of $80 \mathrm{MHz}$ at $800 \mathrm{~nm}$ was used, and the photon fluxes of the relevant excitonic-lines (exciton and two trion states) were summed up to $(12.5 \pm 0.5) \mathrm{kHz}$, corrected by the setup efficiency resulting in a photon flux of $(0.45 \pm 0.05) \mathrm{MHz}$ and divided by the excitation rate $(80 \mathrm{MHz})$. This results in an overall efficiency of $0.56 \% \pm 0.07 \%$. This value could presumably be significantly increased by optimizing the photon-extraction efficiency of the QDmicrolens used. This becomes clear when the outcoupling efficiency of the pure QD-microlens is calculated from the fiber-collection efficiency $(22 \%)$ and the overall efficiency $(0.56 \%)$. This results in a value of $3 \%$, which is significantly lower than the previously achieved values. $^{31}$ It indicates that the QD is either not integrated in the center of the microlens and/or that it suffers from low internal quantum efficiency.

Figure 2(c) depicts in red a time-resolved measurement of the lifetime of the trion-transition of the fiber-coupled sample, which can be fitted using an exponential decay function to quantify the decay time $\tau_{1}=(0.92 \pm 0.03) \mathrm{ns}$. The result is in quantitative agreement with the value $\left(\tau_{1}=(0.92 \pm 0.02) \mathrm{ns}\right)$ recorded on the free-space configuration before fiber-coupling and shows that fiber-coupling does not influence the lifetime of the QD-microlens.

The emission of the trion line is spectrally filtered by a monochromator and coupled at the monochromator exit slit into a fiber-based HBT setup for coincidence measurements [see Fig. 1(f)]. The off-resonant excitation power under $\mathrm{cw}$ excitation was chosen to saturate the trion line [see Fig. 2(b)]. In Fig. 3(a) the measured second-order photon autocorrelation function is plotted showing clear antibunching at $\tau=0$ ns. A physical description of the measurement is possible by a two-sided exponential function,

$$
g^{(2)}(\tau)=1-\left(\left(1-g^{(2)}(0)\right) e^{-\frac{|\tau|}{\tau_{\mathrm{sp}}}}\right)
$$

The fitting of the equation to the measurement data yields $g^{(2)}(0)$ $=0.00 \pm_{0.00}^{0.04}$, which verifies a very low probability of multi-photon emission events. Noteworthy, the spontaneous decay time of $\tau_{\mathrm{sp}}=(0.93 \pm 0.05) \mathrm{ns}$ resulting from this fit is in very good agreement with the lifetime measurement shown in Fig. 2(c).

Furthermore, the quantum nature of emission was also characterized under the more application relevant pulsed excitation. For this purpose, the wavelength of a tunable titanium-sapphire laser was set to $865 \mathrm{~nm}$, i.e., resonant with the wetting layer, and the excitation power was reduced until the detected count rate of the trion line had halved to reduce the contribution of uncorrelated background emission, which increases in relative strength with the excitation power. Figure 3(b) displays the corresponding correlation histogram.

To determine the $g^{(2)}(0)$-value, we fitted the experimental data with a sequence of equidistant photon pulses, each represented by the convolution of a two-sided exponential decay $\left(\tau_{1}=0.92 \mathrm{~ns}\right)$ with a Gaussian function of 495 ps width (full width at half maximum), considering the time resolution of the HBT setup. Assuming a constant area A of the finite-time-delay pulses, the $g^{(2)}(0)$-value is expressed by the ratio $A_{0} / A$, where $A_{0}$ corresponds to the area of the zero-time-delay peak. This evaluation results in $g^{(2)}(0)=0.13 \pm 0.05$.
The differences in the $g^{(2)}(0)$-values under $\mathrm{cw}$ and pulsed excitation can presumably be attributed to charge-carrier recapture processes in the case of pulsed excitation, ${ }^{48}$ which can no longer be neglected and, in addition, the autocorrelation of the laser using the HBT-setup indicated that the laser pulses of lower intensity were also observed outside the expected time windows given by the repetition rate. Nevertheless, the device fulfills the requirements of a fiber-coupled pure single photon source on demand, whereby the slight increase of multi-photon events in the case of pulsed excitation compared to $\mathrm{cw}$ excitation could certainly be improved by resonant excitation schemes. The results of another fiber-coupled sample are given in the supplementary material. The device shows very comparable performance in terms of count rates, coupling efficiency, and multi-photon suppression, which underlines the high-reproducibility of our approach. In fact, as detailed in the supplementary material, the biexciton emission shows a measured count rate of $111 \mathrm{kHz}(3.9 \mathrm{MHz}$ at the end of the fiber, fiber-coupling efficiency: $24 \% \pm 2 \%$ ) in saturation, while the exciton line has a count rate of $48 \mathrm{kHz}$ (corrected: 1.7 $\mathrm{MHz})$ under $\mathrm{cw}$ excitation, and a $\mathrm{g}^{(2)}(0)$ of $7 \% \pm 2 \%$ under pulsed excitation.

\section{CONCLUSION}

In summary, we realized a user-friendly single-mode fibercoupled single-photon source with excellent optical and quantum optical properties. The device fabrication benefits from the synergetic combination of 3D electron-beam lithography and femtosecond $3 \mathrm{D}$ direct laser writing, which enables us to join the advantages of both methods and to realize a robust, single-mode fiber coupled micro-optical on-chip system based on a single preselected semiconductor QD. Explicitly, a QD-microlens produced using a $3 \mathrm{D}$ in situ technique was combined with a total-internalreflection micro-objective using 3D direct laser writing. In addition, a fiber chuck was written with sub-micrometer alignment accuracy onto the deterministically fabricated QD-microlens, which allows a SMF with a $3 \mathrm{D}$ printed incoupling lens on its facet to be inserted and permanently attached to the QD-microlens-microobjective assembly. A coupling efficiency of $22 \%$ was determined for the fiber-coupled source, leading to a measured count rate of $42 \mathrm{kHz}(8.9 \mathrm{kHz})$ under $\mathrm{cw}$ (pulsed) optical excitation from which we deduce a maximum single-photon rate of $1.5 \mathrm{MHz}(0.3 \mathrm{MHz})$ at the output of the fiber by considering the setup efficiency $(2.8 \%)$. This technology concept has a high potential to pave the way for the single-mode coupled stand-alone single-photon sources of a high emission rate and quantum optical quality in the future, as demanded with regard to the implementation of scalable quantum networks.

\section{SUPPLEMENTARY MATERIAL}

See the supplementary material for a discussion on the choice of the solid immersion lens shape on top of single-QD devices and its implications for improving collection efficiency. In addition, the optical data are presented on a second fiber-coupled QD microlens to demonstrate the reproducibility of the developed fiber-coupling concept. 


\section{ACKNOWLEDGMENTS}

S.R. acknowledges funding from the German Federal Ministry of Education and Research (BMBF) through the Project No. Q.Link.X, the German Research Foundation via the Project No. Re2974/10-1, and from the Project Nos. EMPIR, 14IND05, MIQC2, and SIQUST (the EMPIR initiative is co-funded by the European Union's Horizon 2020 research and innovation program and the EMPIR Participating States). H.G., K.W., S.T., and A.H. acknowledge funding by ERC (AdG ComplexPlas, PoC 3DPrintedoptics), BMBF (Q.Link.X, Printoptics, Printfunction), MWK BW (ZAQuant), BW Stiftung (Opterial) and DFG (Grant Nos. SPP1839 and 1929). We thank the Physikalisch-Technische Bundesanstalt Berlin for technical support. S.L.P., M.S., and P.M. greatly acknowledge funding from the BMBF (German Federal Ministry of Education and Research) via the Project No. Q.Link.X (Grant No. 16KIS0862) and support via the Project No. EMPIR 17FUN06 SIQUST. This project received funding from the EMPIR program cofinanced by the Participating States and from the European Union's Horizon 2020 research and innovation program.

\section{DATA AVAILABILITY}

The data that support the findings of this study are available from the corresponding author upon reasonable request.

\section{REFERENCES}

${ }^{1}$ L.-M. Duan, M. D. Lukin, J. I. Cirac, and P. Zoller, Nature 414, 413 (2001).

${ }^{2}$ N. Gisin and R. Thew, Nat. Photonics 1, 165 (2007).

${ }^{3}$ C. H. Bennett and G. Brassard, in Proceedings of IEEE International Conference on Computers, Systems and Signal Processing (IEEE, 1984), Vol. 175.

${ }^{4}$ N. Gisin, G. Ribordy, W. Tittel, and H. Zbinden, Rev. Mod. Phys. 74, 145 (2002).

${ }^{5}$ B. Fröhlich, M. Lucamarini, J. F. Dynes, L. C. Comandar, W. W.-S. Tam, A. Plews, A. W. Sharpe, Z. Yuan, and A. J. Shields, Optica 4, 163 (2017).

${ }^{6}$ A. Boaron, G. Boso, D. Rusca, C. Vulliez, C. Autebert, M. Caloz, M. Perrenoud, G. Gras, F. Bussières, M.-J. Li, D. Nolan, A. Martin, and H. Zbinden, Phys. Rev. Lett. 121, 190502 (2018).

${ }^{7}$ R. Ursin, F. Tiefenbacher, T. Schmitt-Manderbach, H. Weier, T. Scheidl, M. Lindenthal, B. Blauensteiner, T. Jennewein, J. Perdigues, P. Trojek, B. Ömer, M. Fürst, M. Meyenburg, J. Rarity, Z. Sodnik, C. Barbieri, H. Weinfurter, and A. Zeilinger, Nat. Phys. 3, 481 (2007).

${ }^{8}$ S.-K. Liao, H.-L. Yong, C. Liu, G.-L. Shentu, D.-D. Li, J. Lin, H. Dai, S.-Q. Zhao, B. Li, J.-Y. Guan, W. Chen, Y.-H. Gong, Y. Li, Z.-H. Lin, G.-S. Pan, J. S. Pelc, M. M. Fejer, W.-Z. Zhang, W.-Y. Liu, J. Yin, J.-G. Ren, X.-B. Wang, Q. Zhang, C.-Z. Peng, and J.-W. Pan, Nat. Photonics 11, 509 (2017).

${ }^{9}$ G. Vallone, D. Bacco, D. Dequal, S. Gaiarin, V. Luceri, G. Bianco, and P. Villoresi, Phys. Rev. Lett. 115, 040502 (2015).

${ }^{10}$ S.-K. Liao, W.-Q. Cai, W.-Y. Liu, L. Zhang, Y. Li, J.-G. Ren, J. Yin, Q. Shen, Y. Cao, Z.-P. Li, F.-Z. Li, X.-W. Chen, L.-H. Sun, J.-J. Jia, J.-C. Wu, X.-J. Jiang, J.-F. Wang, Y.-M. Huang, Q. Wang, Y.-L. Zhou, L. Deng, T. Xi, L. Ma, T. Hu, Q. Zhang, Y.-A. Chen, N.-L. Liu, X.-B. Wang, Z.-C. Zhu, C.-Y. Lu, R. Shu, C.-Z. Peng, J.-Y. Wang, and J.-W. Pan, Nature 549, 43 (2017).

${ }^{11}$ W.-Y. Hwang, Phys. Rev. Lett. 91, 057901 (2003).

${ }^{12}$ H.-K. Lo, X. Ma, and K. Chen, Phys. Rev. Lett. 94, 230504 (2005).

${ }^{13}$ L. Schweickert, K. D. Jöns, K. D. Zeuner, S. F. Covre da Silva, H. Huang, T. Lettner, M. Reindl, J. Zichi, R. Trotta, A. Rastelli, and V. Zwiller, Appl. Phys. Lett. 112, 093106 (2018).

${ }^{14}$ L. Hanschke, K. A. Fischer, S. Appel, D. Lukin, J. Wierzbowski, S. Sun, R. Trivedi, J. Vučković, J. J. Finley, and K. Müller, npj Quantum Inf. 4, 1 (2018).

${ }^{15}$ H.-K. Lo, M. Curty, and K. Tamaki, Nat. Photonics 8, 595 (2014).

${ }^{16}$ X.-B. Wang, Phys. Rev. Lett. 94, 230503 (2005).
${ }^{17}$ H.-J. Briegel, W. Dür, J. I. Cirac, and P. Zoller, Phys. Rev. Lett. 81, 5932 (1998).

${ }^{18}$ N. Somaschi, V. Giesz, L. De Santis, J. C. Loredo, M. P. Almeida, G. Hornecker, S. L. Portalupi, T. Grange, C. Antón, J. Demory, C. Gómez, I. Sagnes, N. D. Lanzillotti-Kimura, A. Lemaítre, A. Auffeves, A. G. White, L. Lanco, and P. Senellart, Nat. Photonics 10, 340 (2016).

${ }^{19}$ A. Thoma, P. Schnauber, M. Gschrey, M. Seifried, J. Wolters, J.-H. Schulze, A. Strittmatter, S. Rodt, A. Carmele, A. Knorr, T. Heindel, and S. Reitzenstein, Phys. Rev. Lett. 116, 033601 (2016).

${ }^{20}$ H. Wang, H. Hu, T.-H. Chung, J. Qin, X. Yang, J.-P. Li, R.-Z. Liu, H.-S. Zhong, Y.-M. He, X. Ding, Y.-H. Deng, Q. Dai, Y.-H. Huo, S. Höfling, C.-Y. Lu, and J.-W. Pan, Phys. Rev. Lett. 122, 113602 (2019).

${ }^{21}$ J. Liu, R. Su, Y. Wei, B. Yao, S. F. C. d. Silva, Y. Yu, J. Iles-Smith, K. Srinivasan, A. Rastelli, J. Li, and X. Wang, Nat. Nanotechnol. 14, 586 (2019).

${ }^{22}$ G. Bulgarini, M. E. Reimer, M. Bouwes Bavinck, K. D. Jöns, D. Dalacu, P. J. Poole, E. P. A. M. Bakkers, and V. Zwiller, Nano Lett. 14, 4102 (2014).

${ }^{23}$ A. Schlehahn, S. Fischbach, R. Schmidt, A. Kaganskiy, A. Strittmatter, S. Rodt, T. Heindel, and S. Reitzenstein, Sci. Rep. 8, 1340 (2018).

${ }^{24} \mathrm{X}$. Xu, F. Brossard, K. Hammura, D. A. Williams, B. Alloing, L. H. Li, and A. Fiore, Appl. Phys. Lett. 93, 021124 (2008).

${ }^{25}$ H. Snijders, J. A. Frey, J. Norman, V. P. Post, A. C. Gossard, J. E. Bowers, M. P. van Exter, W. Löffler, and D. Bouwmeester, Phys. Rev. Appl. 9, 031002 (2018).

${ }^{26}$ A. Musiał, K. Żołnacz, N. Srocka, O. Kravets, J. Große, J. Olszewski, K. Poturaj, G. Wójcik, P. Mergo, K. Dybka, M. Dyrkacz, M. Dłubek, K. Lauritsen, A. Bülter, P.-I. Schneider, L. Zschiedrich, S. Burger, S. Rodt, W. Urbańczyk, G. Sęk,, and S. Reitzenstein, Adv. Quantum Technol. 3, 2000018 (2020).

${ }^{27}$ C.-M. Lee, M. A. Buyukkaya, S. Aghaeimeibodi, A. Karasahin, C. J. K. Richardson, and E. Waks, Appl. Phys. Lett. 114, 171101 (2019).

${ }^{28}$ R. S. Daveau, K. C. Balram, T. Pregnolato, J. Liu, E. H. Lee, J. D. Song, V. Verma, R. Mirin, S. W. Nam, L. Midolo, S. Stobbe, K. Srinivasan, and P. Lodahl, Optica 4, 178 (2017).

${ }^{29}$ N. Montaut, L. Sansoni, E. Meyer-Scott, R. Ricken, V. Quiring, H. Herrmann, and C. Silberhorn, Phys. Rev. Appl. 8, 024021 (2017).

${ }^{30}$ M. Schwartz, E. Schmidt, U. Rengstl, F. Hornung, S. Hepp, S. L. Portalupi, K. Ilin, M. Jetter, M. Siegel, and P. Michler, Nano Lett. 18, 6892 (2018).

${ }^{31}$ M. Gschrey, A. Thoma, P. Schnauber, M. Seifried, R. Schmidt, B. Wohlfeil, L. Krüger, J. H. Schulze, T. Heindel, S. Burger, F. Schmidt, A. Strittmatter, S. Rodt, and S. Reitzenstein, Nat. Commun. 6, 7662 (2015).

${ }^{32}$ A. W. Schell, J. Kaschke, J. Fischer, R. Henze, J. Wolters, M. Wegener, and O. Benson, Sci. Rep. 3, 1577 (2013).

${ }^{33}$ T. Gissibl, S. Thiele, A. Herkommer, and H. Giessen, Nat. Photonics 10, 554 (2016).

${ }^{34}$ T. Gissibl, S. Thiele, A. Herkommer, and H. Giessen, Nat. Commun. 7, 11763 (2016).

${ }^{35}$ M. Sartison, S. L. Portalupi, T. Gissibl, M. Jetter, H. Giessen, and P. Michler, Sci. Rep. 7, 039916 (2017).

${ }^{36}$ A. Bogucki, Ł. Zinkiewicz, M. Grzeszczyk, W. Pacuski, K. Nogajewski, T. Kazimierczuk, A. Rodek, J. Suffczyński, K. Watanabe, T. Taniguchi, P. Wasylczyk, M. Potemski, and P. Kossacki, Light: Sci. Appl. 9, 48 (2020).

${ }^{37}$ S. Fischbach, A. Schlehahn, A. Thoma, N. Srocka, T. Gissibl, S. Ristok, S. Thiele, A. Kaganskiy, A. Strittmatter, T. Heindel, S. Rodt, A. Herkommer, H. Giessen, and S. Reitzenstein, ACS Photonics 4, 1327 (2017).

${ }^{38}$ M. Sartison, K. Weber, S. Thiele, L. Bremer, S. Fischbach, T. Herzog, S. Kolatschek, S. Reitzenstein, A. Herkommer, P. Michler, S. L. Portalupi, and H. Giessen, arXiv:2007.06369 (2020).

${ }^{39}$ A. Bogucki, Ł. Zinkiewicz, W. Pacuski, P. Wasylczyk, and P. Kossacki, Opt. Express 26, 011513 (2018).

${ }^{40}$ P.-I. Dietrich, M. Blaicher, I. Reuter, M. Billah, T. Hoose, A. Hofmann, C. Caer, R. Dangel, B. Offrein, U. Troppenz, M. Moehrle, W. Freude, and C. Koos, Nat. Photonics 12, 241 (2018).

${ }^{41}$ A. Schlehahn, M. Gaafar, M. Vaupel, M. Gschrey, P. Schnauber, J.-H. Schulze, S. Rodt, A. Strittmatter, W. Stolz, A. Rahimi-Iman, T. Heindel, M. Koch, and S. Reitzenstein, Appl. Phys. Lett. 107, 041105 (2015). 
${ }^{42}$ A. Kaganskiy, T. Heuser, R. Schmidt, S. Rodt, and S. Reitzenstein, J. Vac. Sci. Technol., B 34, 061603 (2016).

${ }^{43}$ M. Sartison, S. Seyfferle, S. Kolatschek, S. Hepp, M. Jetter, P. Michler, and S. L. Portalupi, Appl. Phys. Lett. 114, 222101 (2019).

${ }^{44}$ S. Kolatschek, S. Hepp, M. Sartison, M. Jetter, P. Michler, and S. L. Portalupi, J. Appl. Phys. 125, 045701 (2019).
${ }^{45}$ M. Schmid, D. Ludescher, and H. Giessen, Opt. Mater. Express 9, 4564 (2019).

${ }^{46} \mathrm{~K}$. Weber, F. Hütt, S. Thiele, T. Gissibl, A. Herkommer, and H. Giessen, Opt. Express 25, 019672 (2017).

${ }^{47}$ L. Rickert, T. Kupko, S. Rodt, S. Reitzenstein, and T. Heindel, Opt. Express 27, 036824 (2019).

${ }^{48}$ T. Aichele, V. Zwiller, and O. Benson, New J. Phys. 6, 90 (2004). 